INPLASY

PROTOCOL

To cite: Li et al.

Polyetheretherketone(PEEK) rods versus titanium rods for lumbar fusion: a meta-analysis. Inplasy protocol 2021110049. doi:

10.37766/inplasy2021.11.0049

Received: 15 November 2021

Published: 15 November 2021

Corresponding author:

Wenhao Li

18800152886@163.com

Author Affiliation:

Dongzhimen Hospital Affiliated

to Beijing University of

Chinese Medicine.

Support: No financial support.

Review Stage at time of this

submission: Data analysis.

Conflicts of interest:

None declared.

\section{Polyetheretherketone(PEEK) rods versus titanium rods for lumbar fusion: a meta-analysis}

Li, WH1; Niu, YQ2; Zhao, H33 Li, CH4; Yang, KT5; Liu, T6; Guan, JB7; Zhong, WQ8; Yang, YD9; Yu, $\mathrm{X}^{10}$.

Review question / Objective: We conducted this meta-analysis to systematically compare the effects of the PEEK rod and titanium rod in lumbar fusion surgery.

Condition being studied: The effectiveness and safety of the PEEK rod have been verified by a large number of biomechanical studies and clinical researches, including some randomized controlled trials.

Information sources: All studies included in this meta-analysis met the following criteria: (1) published clinical RCT; (2) patients who have undergone lumbar fusion due to degenerative diseases of the lumbar spine, and the age, gender, and nationality were not limited; and (3) PEEK rodpedicle screw fixation was used as an intervention measure, and titanium rod-pedicle screw fixation was used as a control measure, and complete comparison data between two groups could be obtained.

INPLASY registration number: This protocol was registered with the International Platform of Registered Systematic Review and Meta-Analysis Protocols (INPLASY) on 15 November 2021 and was last updated on 15 November 2021 (registration number INPLASY2021110049).

\section{INTRODUCTION}

Review question / Objective: We conducted this meta-analysis to systematically compare the effects of the PEEK rod and titanium rod in lumbar fusion surgery.
Condition being studied: The effectiveness and safety of the PEEK rod have been verified by a large number of biomechanical studies and clinical 
researches, including some randomized controlled trials.

\section{METHODS}

Participant or population: Patients who have undergone lumbar fusion due to degenerative diseases of the lumbar spine, the age, gender, and nationality were notlimited.

Intervention: PEEK rod-pedicle screw fixation + bone graft fusion.

Comparator: Titanium rod-pedicle screw fixation + bone graft fusion.

Study designs to be included: Randomized controlled trials.

Eligibility criteria: All studies included in this meta-analysis met the following criteria: (1) published clinical RCT; (2) patients who have undergone lumbar fusion due to degenerative diseases of the lumbar spine, and the age, gender, and nationality were not limited; and (3) PEEK rod-pedicle screw fixation was used as an intervention measure, and titanium rodpedicle screw fixation was used as a control measure, and complete comparison data between two groups could be obtained.

Information sources: A systematic computer-based retrieval was performed on the literatures published before September 1, 2021, in PubMed, Embase, Cochrane Library, China National Knowledge Infrastructure database (CNKI), WanFang database, and Chinese Scientific Journal Database. At the same time, we traced the references of the included literatures and the meta-analysis related to this research, screened, and evaluated the references to determine potential researches.

Main outcome(s): A total of 255 related studies were confirmed from the electronic database. After deleting duplicate studies, 178 studies were obtained. After careful full-text evaluation of these studies according to the inclusion and exclusion criteria, 7 RCT studies with 429 patients were included in the final comprehensive analysis.

Quality assessment / Risk of bias analysis: The included 7 studies were high-risk in terms of randomization, allocation concealment, and blinding. None of the 7 studies withdrew or was lost to follow-up, and the data was complete. In terms of selective reporting, since the study of $\mathrm{Li}$ et al. did not report data on the index of bone graft fusion rate, it was high risk, and the remaining studies were low risk.

Strategy of data synthesis: The Review Manager software(RevMan 5.3) was used for statistical analysis. Continuous variables were reported as mean difference(MD) and $95 \%$ confidence interval(Cl), while dichotomy variables were reported as odds ratio(OR) and $95 \% \mathrm{Cl}$. Statistical heterogeneity was judged by the combination of $Q$ value statistics and 12 statistics. The larger the 12, the greater the heterogeneity. If there was heterogeneity in the study $(12 \geq 50 \%)$, the random effects model was adopted; otherwise, the fixed effects model was adopted( $12<50 \%)$. The extracted data was input into the computer, reviewed, and independently analyzed by two researchers.

Subgroup analysis: This study did not perform subgroup analysis.

Sensitivity analysis: For ODI score (12=87\%) and VAS score for LBP $(12=94 \%)$, the sensitivity was tested by eliminating the literature one by one, and found after excluding the study of Li et al., the 12 and $P$ values of the VAS score for LBP did not change much, while the heterogeneity of the ODI score became significantly smaller $(12=0 \%)$, and the $P$ value decreased from 0.01 to 0.0004 .

Language: Lumbar fusion, PEEK rod, Titanium rod, Meta-analysis.

Country(ies) involved: China. 
Keywords: Lumbar fusion, PEEK rod, Titanium rod, Meta-analysis

Contributions of each author:

Author 1 - Wenhao Li.

Author 2 - Yiqun Niu.

Author 3 - He Zhao.

Author 4 - Chuanhong Li.

Author 5 - Kaitan Yang.

Author 6 - Tao Liu.

Author 7 - Jianbin Guan.

Author 8 - Wenqing Zhong.

Author 9 - Yongdong Yang.

Author 10 - Xing Yu. 